\title{
Mycoplasma pneumoniae pneumonia with pulmonary embolism: A study on pediatric cases in Jilin province of China
}

\author{
CHU-QIAO SHENG, CHUN-FENG YANG, YU AO, ZHI-YUE ZHAO and YU-MEI LI \\ Department of Pediatric Intensive Care Unit, The First Hospital of Jilin University, Changchun, Jilin 130021, P.R. China
}

Received February 13, 2020; Accepted November 26, 2020

DOI: $10.3892 /$ etm. 2021.9634

\begin{abstract}
Mycoplasma is one of the most common pathogens causing community-acquired pneumonia in pediatric patients. In recent years, the number of refractory or severe cases with drug resistance has been gradually increasing and cases that developed embolism after Mycoplasma pneumoniae (M. pneumoniae) infection have been reported. The present study retrospectively analyzed the clinical features, diagnosis and treatment of $M$. pneumoniae pneumonia (MPP) combined with pulmonary embolism (PE) in a series of 7 cases encountered between January 1st, 2016 to August 1st, 2019 at the Department of Pediatric Intensive Care Unit of The First Hospital of Jilin University (Changchun, China). Combined with relevant Chinese and international studies published during the last two decades, a comprehensive analysis was performed. All of the pediatric patients of the present study had fever, cough and dyspnea respiratory symptoms at onset and the disease progressed rapidly. Thereafter, PE was confirmed by a series of examinations. Pulmonary CT indicated patchy inflammations and significantly elevated D-dimer levels, accompanied by positive anticardiolipin antibodies. Furthermore, a filling defect in the pulmonary artery branch was observed on CT pulmonary angiography (CTPA) examination. In 2 cases, the condition was improved with anti-infection and anticoagulation treatment with low-molecular-weight heparin and warfarin, respectively, and the pulmonary embolism disappeared after 3-4 months. A total of 5 cases, who were not responsive to the drug treatment, underwent surgical resection.
\end{abstract}

Correspondence to: Professor Yu-Mei Li, Department of Pediatric Intensive Care Unit, The First Hospital of Jilin University, 1 Xinmin Street, Changchun, Jilin 130021, P.R. China

E-mail: liyumei201912@126.com

Abbreviations: ACA, anticardiolipin antibody; CTPA, computed tomographic pulmonary angiography; M. pneumoniae, Mycoplasma pneumoniae; MPP, M. pneumoniae pneumonia; PE, pulmonary embolism

Key words: mycoplasma pneumonia, pediatric patients, pulmonary embolism, refractory mycoplasma pneumonia, computed tomographic pulmonary angiography
During the operation, the local tissues were determined to be infarcted and the pathological diagnosis was consistent with pulmonary infarction. Among the 5 cases, 2 died of Acute Respiratory Distress Syndrome at 3-8 days after the operation. The remaining patients underwent 6-12 months of follow-up and respiratory rehabilitation and their quality of life is now good. In conclusion, compared with healthy individuals, pediatric patients with critical MPP have an elevated risk of embolism. It is necessary to be vigilant regarding whether MMP is combined with PE and perform timely CTPA examination. Early detection, early treatment and surgical intervention (if necessary) may significantly reduce the risk of mortality and disability.

\section{Introduction}

Mycoplasma pneumoniae ( $M$. pneumoniae) is the most common pathogen in pediatric patients with community-acquired pneumonia $(1,2)$ which breaks out every 3-5 years (3). In recent years, the number of refractory or severe cases with drug resistance has been gradually increasing (4-6). In addition to pulmonary inflammation as the most common manifestation, $M$. pneumoniae infection may also cause damage to multiple systems and organs (7-9). In the last 20 years, $\sim 60$ cases of $M$. pneumoniae infection with thrombotic disease in pediatric patients have been reported worldwide (10-15). The present study reported on a series of 7 cases of $M$. pneumoniae pneumonia (MPP) accompanied by pulmonary embolism (PE) encountered January 1st, 2016 to August 1st, 2019 at the Department of Pediatric Intensive Care Unit of The First Hospital of Jilin University (Changchun, China), and the clinical data of these cases were reviewed. The present study aimed to improve the understanding of clinicians regarding the laboratory examinations, diagnosis and treatments for pediatric patients with MPP-associated PE.

\section{Case report}

Cases. MPP-associated PE was confirmed in 7 cases by radiological examination combined with serological tests and the corresponding clinical data were collected and summarized. The present study had been approved by the Ethics Committee of the First Hospital of Jilin University (Changchun, China; approval no. 2019-253). 
Baseline data. The seven cases were aged between 6 and 11 years (median, 8.0 years) with a male/female ratio of $4: 3$, as presented in Table I. All patients were otherwise physically healthy. Patients with a family history of thrombophilia and a history of allergy were excluded.

Clinical symptoms and physical signs. All of the patients had a cough and fever as the initial symptoms, typically irritable cough with viscous sputum, and remittent fever. Among them, 5 cases developed dyspnea within 2 to 6 days and were hospitalized on day 3-12 from onset. These cases developed PE on day 10-14 and their condition soon deteriorated. Older pediatric patients complained of chest pain, chest tightness or sudden dyspnea; younger patients were unable to describe their symptoms, but physical examinations revealed spiritlessness, aggravating dyspnea, flapping of nasal wings, reduced respiratory movement amplitude on the affected side and weak breath sounds. One case was combined with swelling in the right lower limb.

\section{Results of auxiliary examinations}

Laboratory tests. The serum biochemistry results of the cases are presented in Table II. The serum M. pneumoniae antibody titers (Particle Agglutination assay; SERODIA-MYCOII; Fujirebio) were increased by varying degrees [from negative titer at presentation to positive titer at the 2nd examination $(n=1)$ or $>4$-fold increased antibody titers during admission $(n=6)]$. All cases had a significant increase in the platelet count and D-dimer level. The levels of protein $\mathrm{C}$ and protein $\mathrm{S}$ first transiently decreased and were then restored to normal. Furthermore, two cases were weakly positive for anticardiolipin antibody (ACA). In addition, four cases were positive for ACA as detected by ELISA (16) (QUANTA Lite ACA IgG III; Inova). A total of four cases received bronchoscopy, through which endobronchitis and necrotizing pneumonia were revealed. The bronchoalveolar lavage fluid was tested positive for M. pneumoniae (DNA sequence copy number, $7,880-16,343)$ and positivity for the macrolide resistance gene was detected in 2 cases.

Radiological examination. All 7 cases received dynamic monitoring by chest X-ray, pulmonary CT scan and computed tomographic pulmonary angiography (CTPA) during hospitalization. Furthermore, 6 cases were indicated to have extensive diffuse inflammatory changes in the two lungs upon chest X-ray or pulmonary CT scan (Figs. 1 and 2) and 1 case had subcutaneous emphysema (Fig. 3). Furthermore, 4 cases were combined with a moderate amount of pleural effusion and 1 case was combined with mild pericardial effusion (Fig. 4). CTPA indicated that 2 cases had a pulmonary arterial embolism in multiple branches bilaterally (Fig. 5, arrows); 2 cases had a pulmonary arterial embolism in the upper lobe of the right lung and one of the two lesions was located at the distal end of the right upper lung; 1 case had filling defects in the pulmonary artery branches in the upper lobe of the left lung; 2 cases had distal pulmonary artery embolism in the lower lobe of the right lung. Furthermore, 1 case with swelling in the lower limbs received local vascular ultrasound examination, through which thrombosis in the common femoral vein was detected.
Table I. Baseline demographic and clinical characteristics of the patients $(n=7)$.

\begin{tabular}{lc}
\hline Characteristic & Value \\
\hline Demographics & \\
Age (years) & $8(6,11)$ \\
Male sex & $4(57.14)$ \\
Anthropometry & \\
Body weight $(\mathrm{kg})$ & $24.2(21.3,30)$ \\
Body height (cm) & $123.4(119.5,130)$ \\
BMI z-score & $0.5(-0.5,1)$ \\
Clinical symptoms & \\
Cough & $7(100)$ \\
Fever & $7(100)$ \\
Dyspnea & $5(71.43)$ \\
Swelling in limb & $1(14.29)$ \\
Radiological examination & \\
Pulmonary CT & \\
Extensive diffuse inflammatory & $6(85.71)$ \\
Subcutaneous emphysema & $1(14.29)$ \\
Pleural effusion & $4(57.14)$ \\
Pericardial effusion & $1(14.29)$ \\
Pulmonary arterial embolism & \\
Bilateral multiple branches & $2(28.57)$ \\
Upper lobe of the right lung & $2(28.57)$ \\
Lower lobe of the right lung & $2(28.57)$ \\
Upper lobe of the left lung & $1(14.29)$ \\
\hline
\end{tabular}

Values are expressed as the median (interquartile range) or $\mathrm{n}(\%)$. BMI, body mass index.

Treatment. After admission, all of the cases were given the standard anti-infection therapy of macrolides and certain patients received concurrent antibiotic therapy with third-generation cephalosporins or carbapenems. Over the same period, moxifloxacin was selected for the 2 cases with positivity for the drug resistance gene. Those patients with dyspnea were treated by tracheal intubation and mechanical ventilation. In the meantime, other systemic treatments, such as organ protection and nutritional support, were given. Risk stratification was performed based on the guidelines of the American College of Chest Physicians (17), along with the thrombolysis and anti-coagulation therapies. Low-molecular-weight heparin calcium was injected subcutaneously at the dose of $50 \mathrm{IU} / \mathrm{kg}$ per time, twice daily. During the treatment, the pediatric patients were properly immobilized to avoid violent cough and movement. Since these pediatric patients did not have a basic history of congenital heart disease and presented with no pulmonary thrombosis, no shock due to PE and no deep vein thrombosis, thrombophilia was excluded and thrombolytic therapy was therefore not selected. However, anticoagulant therapy had no curative effect in 5 cases and the disease progressed to pulmonary infarction; thus, surgical resections were conducted. 


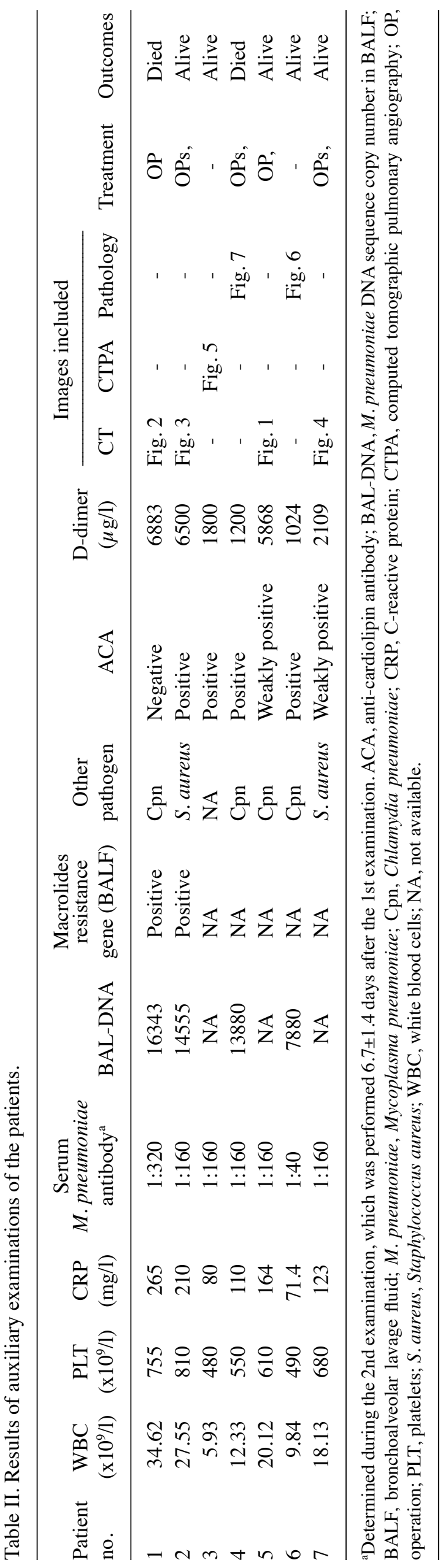

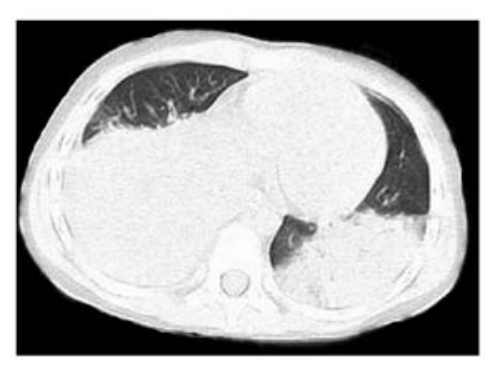

Figure 1. Bilateral pulmonary extensive diffuse inflammation, with bilateral atelectasis of the dorsal lobes of a 7 year old male.

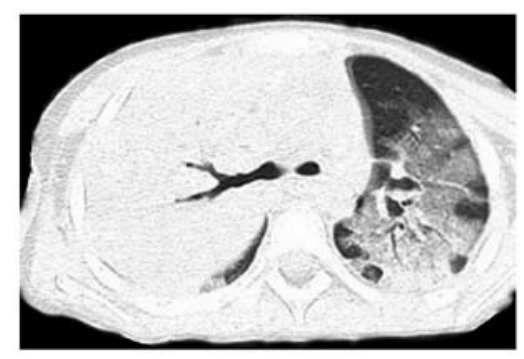

Figure 2. Bilateral pulmonary extensive diffuse inflammation with consolidation in the right lung of an 8 year old male.

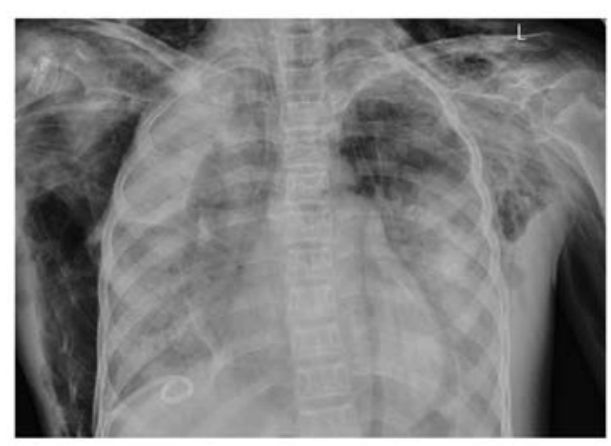

Figure 3. Severe inflammatory changes in the bilateral lungs with extensive subcutaneous emphysema of an 11 year old male.

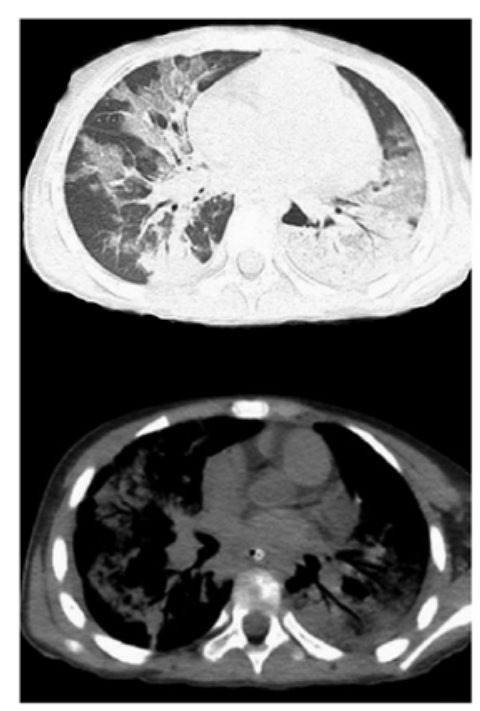

Figure 4. Inflammation in the middle lobe of the right lung and all lobes of the left lung of a 6 year old female, with partial atelectasis in the lower lobe of the left lung (upper panel) and mild pericardial effusion (lower panel). 


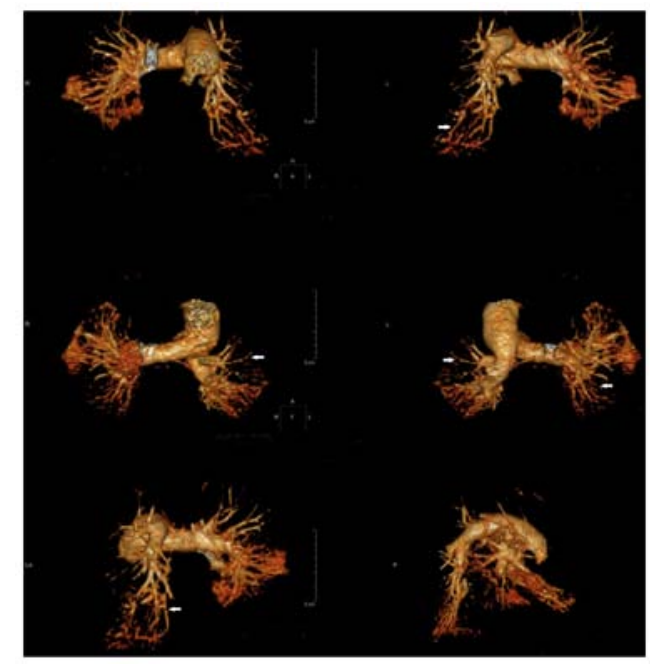

Figure 5. Bilateral pulmonary arterial filling defect of a 6 year old male; arterial embolism is visible in multiple branches, with mild dilation of the pulmonary trunk (white arrows; scale bar, $5 \mathrm{~cm}$ ).

Outcomes and follow-up. Anticoagulant therapy is the first choice for all pediatric patients with PE if there is no contraindication (18). A total of 2 patients achieved significant improvement after 15-21 days of treatment as detected by chest radiological examination and D-dimer test. They were discharged after the symptoms improved, and the PE disappeared 3-5 months later as detected during the follow-up. The 5 remaining cases exhibited no improvement in the local PE, which was confirmed by pulmonary CT scan on day 14-21 during the anticoagulation therapy. Pulmonary infarction was considered in certain patients who had local nodular solid lesions in the lungs with cavitation and the condition deteriorated to high dependence on oxygen. Among them, 2 patients had considerable pleural effusion (pus) and required surgical resection. Intraoperative findings included dark-red resected pulmonary tissues or yellowish-white infarct-like changes. These tissues had no contraction and dilation functions, with high tension and pus coating on their surface. The pathological diagnosis of the resected pulmonary tissues was pulmonary infarction (Figs. 6 and 7). Among them, 2 cases were combined with Acinetobacter baumannii infection after surgery and Acute Respiratory Distress Syndrome occurred 3-8 days later. Although the patients were treated with extracorporeal membrane oxygenation, their condition did not improve and they eventually died. The remaining surviving pediatric patients underwent 6-12 months of follow-up and respiratory rehabilitation and they recovered to a normal state.

Literature search and review. Using 'Pulmonary Embolism' and 'Mycoplasma pneumoniae pneumonia' with 'pediatric' as the keywords, relevant articles were searched in the PubMed, MEDLINE, Update, Web of Science and Embase databases. The Chinese subject heading terms used in the Wanfang, Chinese National Knowledge Infrastructure and Chongqing VIP databases were the same as those above. The inclusion criteria were as follows: i) Pediatric patients with a confirmed diagnosis of MPP and PE during the

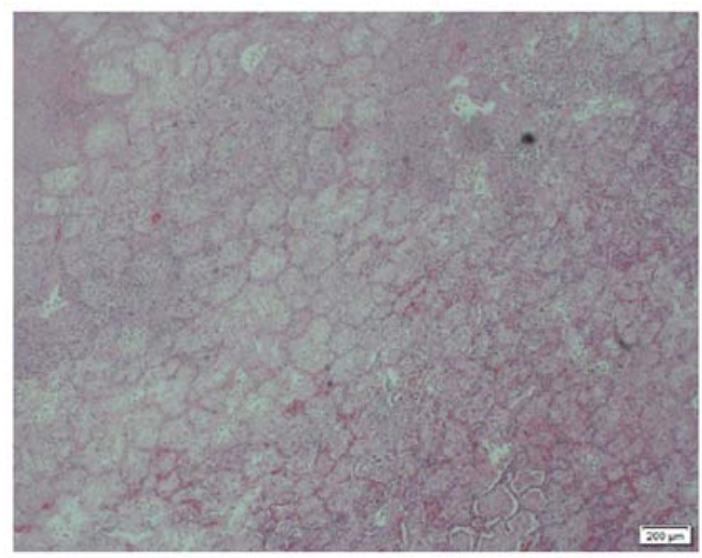

Figure 6. Histological image displaying pulmonary infarction and necrosis with abscess formation in an 8 year old female (H\&E staining; scale bar, $200 \mu \mathrm{m})$.

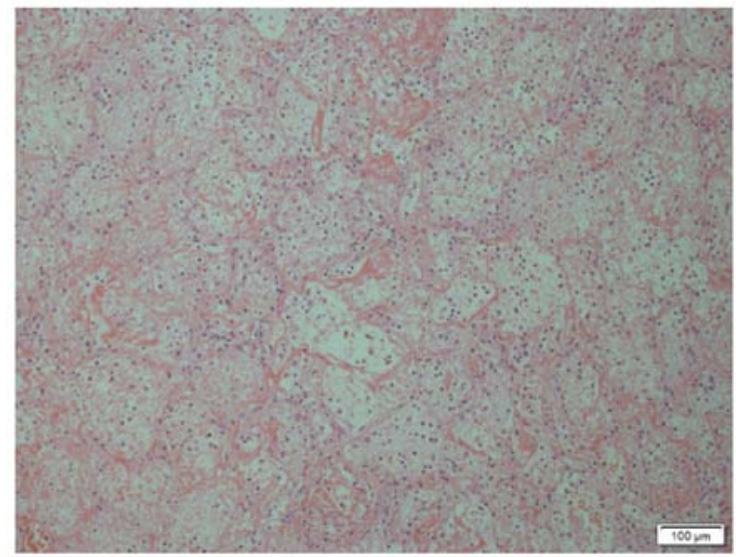

Figure 7. Extensive infarction and necrosis of the resected pulmonary tissues with infiltration of a large amount of inflammatory cells in an 11 year old female (H\&E staining; scale bar, $100 \mu \mathrm{m}$ ).

treatment process; and ii) Studies published within the last 20 years. Exclusion criteria was incomplete clinical information. Ultimately, the clinical data of 10 pediatric cases with MPP and PE were reported and their details are listed in Table III (19-26).

These cases were aged between 6 and 13 years (median age, 9.0 years) with a male/female ratio of 5:1. The levels of $M$. pneumoniae antibody were significantly increased, along with a transient decrease in protein $\mathrm{S}$ and protein $\mathrm{C}$. The lesions were located at the lower lobe close to the hilus of the lung. After receiving anti-infective and anti-coagulant treatments, 8 cases improved but 1 patient died.

\section{Discussion}

Pediatric patients with critical MPP complicated with PE was rarely reported. Cases with mild PE may be asymptomatic, while severe cases may suffer from pulmonary arterial hypertension, unstable hemodynamics or even sudden death $(27,28)$. The common symptoms include shortness of breath, chest pain and even dyspnea (19-26). Missed diagnosis may occur if young pediatric patients are not able to properly describe 


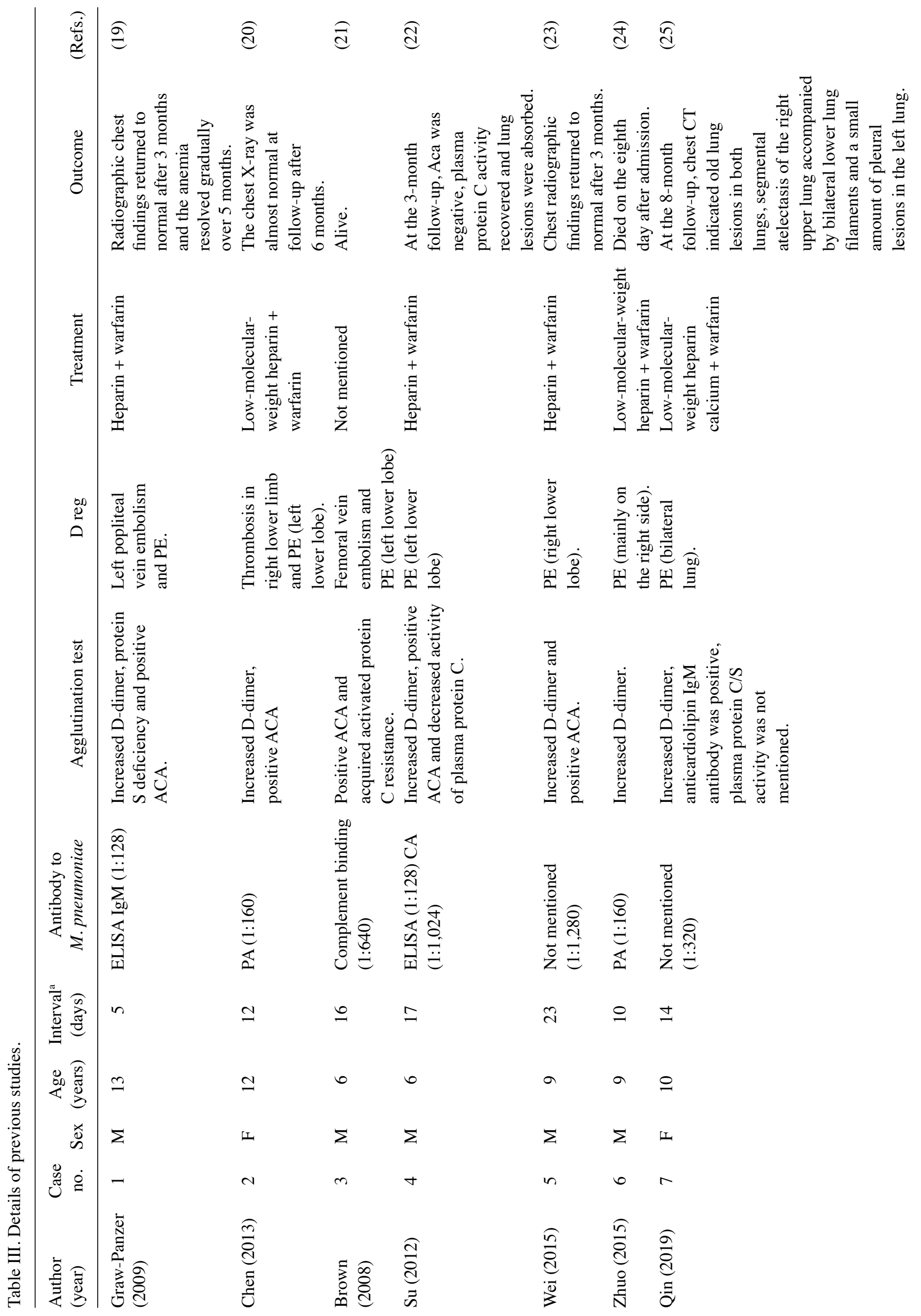


their symptoms. Therefore, if PE is not discovered in a timely manner, the anti-coagulation treatment is delayed and the disease may progress into acute pulmonary infarction or even death. When encountering pediatric cases with MPP, the patient or the parents should be asked whether there is a family history of protein $\mathrm{C} /$ protein $\mathrm{S}$ deficiency, recent history of surgeries or presence of congenital vascular malformation, so as to preliminarily assess the risk of PE. In the present study, evaluation at the early stage of admission indicated a low risk of thrombosis in all cases. However, their symptoms kept on deteriorating during the treatment and chest radiological examination indicated poor recovery. In combination with laboratory tests and chest radiological examination, the diagnosis of PE was confirmed and the standard treatment was provided.

For such pediatric cases, physicians should begin early dynamic monitoring and examination, which may be able to effectively control disease progression, reduce surgical rates and mortality. However, with the current technological standards available, it is still limited to perform the interventional treatment and implement thrombolysis therapy for young pediatric patients with PE. More work, such as a more appropriate design using more sophisticated instruments, still needs to be done to overcome this deficiency in the future.

The pathogenesis of $M$. pneumoniae infection with thrombosis remains to be fully elucidated, but it may be associated with immune damage mediated by infection $(7,29-31)$. Since the membrane proteins and glycolipids of M. pneumoniae have certain common antigens in the heart, liver, lung, brain, kidney and smooth muscle tissues of the human body, upon infection of the host with M. pneumoniae, the corresponding antibodies are produced and the immune complex is formed to activate complement, which produces neutrophils. Previous studies reported that embolism may affect multiple sites of the body after M. pneumoniae infection, including the brain, lower extremity veins, spleen and pulmonary arteries (10-15). Chemokines, which attract a large number of white blood cells to invade the lesion, release a large number of inflammatory mediators and lysosomal enzymes, causing inflammatory damage to target organs. It was reported that patients with MPP and thrombus were positive for ACA $(32,33)$. ACA is an autoantibody that targets antigens in platelets and cardiolipin on endothelial membranes and is associated with thrombogenesis. The present study concluded that $M$. pneumoniae infection may cause vascular endothelial cell injury and ACA positivity, leading to a temporary hypercoagulability state that induces thrombosis. Under severe conditions, M. pneumoniae infection further affects the synthesis of coagulation factors and thrombin (e.g., protein C, protein S and antithrombin III), resulting in embolism. Certain patients may acquire protein $\mathrm{C}$ or protein $\mathrm{S}$ deficiency/resistance $(21,34)$. The D-dimer test is an important screening method for $\mathrm{PE}$ and a negative result may exclude PE with $100 \%$ certainty $(35,36)$. While CTPA is considered as the gold standard for PE diagnosis (37). Based on the above points, in a child with severe MPP, the D-dimer test, ACA test, Protein C test, Protein S test and CTPA should be considered to prevent the occurrence of PE.

At present, the major treatment for pediatric patients with acute PE is anticoagulant therapy, the purpose of which 
is to prevent acute thrombosis and expansion. If available, local thrombus therapy or interventional thrombus therapy may be performed. During the anticoagulation therapy with low-molecular-weight heparin, dynamic monitoring of the coagulation status and treatment outcome is required to avoid bleeding, whose risk is considerable. Furthermore, cooperation between the pediatric thoracic surgery department and the vascular surgery department is preferred and surgical intervention may be provided if necessary.

Critical M. pneumoniae infection in pediatric patients is associated with a high risk of PE. During clinical treatment, such cases should be screened for high-risk factors and patients could be closely monitored for any manifestations of PE. Necessary examinations, particularly CTPA, should be performed to confirm the diagnosis and to initiate standard treatment as early as possible. Surgical intervention is another important salvage to reduce poor prognosis, if the disease progresses to pulmonary infarction, and therefore results in serious and life threatening complications.

\section{Acknowledgements}

Not applicable.

\section{Funding}

No funding was received.

\section{Availability of data}

The datasets used and/or analysed during the current study are available from the corresponding author on reasonable request.

\section{Authors' contributions}

CQS conceived the current study and drafted and revised the manuscript. CFY collected the literature and reviewed and revised the manuscript. YA and ZYZ collected the data and performed initial analyses. YML coordinated and supervised data collection, and critically reviewed the manuscript. CQS and YML confirm the authenticity of all the raw data. All authors read and approved the final manuscript.

\section{Ethics approval and consent to participate}

The research followed international and national regulations in accordance with the Declaration of Helsinki. The study was approved by the Ethics Committee of the First Hospital of Jilin University. (Changchun, China; approval no. 2019-253).

\section{Patient consent for publication}

Written informed consents were obtained from the patients' legal guardians for the publication of any accompanying images prior to submission.

\section{Competing interests}

The authors declare that they have no competing interests.

\section{References}

1. Jain S, Williams DJ, Arnold SR, Ampofo K, Bramley AM, Reed C, Stockmann C, Anderson EJ, Grijalva CG, Self WH, et al: Community-acquired pneumonia requiring hospitalization among U.S. children. N Engl J Med 372: 835-845, 2015.

2. Tashiro M, Fushimi K, Kawano K, Takazono T, Saijo T, Yamamoto K, Kurihara S, Imamura Y, Miyazaki $T$, Yanagihara K, et al: Comparison of efficacy of antimicrobial agents among hospitalized patients with Mycoplasma pneumoniae pneumonia in Japan during large epidemics of macrolide-resistant $M$. pneumoniae infections: A nationwide observational study. Clin Infect Dis 65: 1837-1842, 2017.

3. Omori R, Nakata Y, Tessmer HL, Suzuki S and Shibayama K: The determinant of periodicity in Mycoplasma pneumoniae incidence: An insight from mathematical modelling. Sci Rep 5: 14473, 2015.

4. Tanaka T, Oishi T, Miyata I, Wakabayashi S, Kono M, Ono S, Kato A, Fukuda Y, Saito A, Kondo E, et al: Macrolide-resistant Mycoplasma pneumoniae infection, Japan, 2008-2015. Emerg Infect Dis 23: 1703-1706, 2017.

5. Huang L, Chen H and Peng S: Spontaneous pneumomediastinum, emphysema, and pulmonary bullae associated with refractory Mycoplasma pneumoniae pneumonia in a child. Pediatr Pulmonol 52: E77-E80, 2017.

6. Pereyre S, Goret J and Bébéar C: Mycoplasma pneumoniae: Current knowledge on macrolide resistance and treatment. Front Microbiol 7: 974, 2016.

7. Narita M: Classification of extrapulmonary manifestations due to Mycoplasma pneumoniae infection on the basis of possible pathogenesis. Front Microbiol 7: 23, 2016.

8. Olson D, Watkins LK, Demirjian A, Lin X, Robinson CC, Pretty K, Benitez AJ, Winchell JM, Diaz MH, Miller LA, et al: Outbreak of Mycoplasma pneumoniae-associated Stevens-Johnson syndrome. Pediatrics 136: e386-e394, 2015.

9. Dhaliwal K and Enright K: Rare extrapulmonary complications of Mycoplasma pneumoniae infection. BMJ Case Rep 2016: bcr2015214044, 2016.

10. Witmer CM, Steenhoff AP, Shah SS and Raffini LJ: Mycoplasma pneumoniae, splenic infarct, and transient antiphospholipid antibodies: A new association? Pediatrics 119: e292-e295, 2007.

11. Bao Y, Li X, Wang K, Zhao C, Ji X and Jiang M: Central retinal artery occlusion and cerebral infarction associated with Mycoplasma pneumonia infection in children. BMC Pediatr 16: 210, 2016.

12. Kang B, Kim DH, Hong YJ, Son BK, Lim MK, Choe YH and Kwon YS: Complete occlusion of the right middle cerebral artery associated with Mycoplasma pneumoniae pneumonia. Korean J Pediatr 59: 149-152, 2016.

13. Oeser C, Andreas M, Rath C, Habertheuer A and Kocher A: Left ventricular thrombus in a patient with cutaneous T-cell lymphoma, hypereosinophilia and Mycoplasma pneumoniae infection-a challenging diagnosis: A case report. J Cardiothorac Surg 10: 21, 2015.

14. Bakshi M, Khemani C, Vishwanathan V, Anand RK and Khubchandani RP: Mycoplasma pneumonia with antiphospholipid antibodies and a cardiac thrombus. Lupus 15: 105-106, 2006.

15. Jin X, Zou Y, Zhai J, Liu J and Huang B: Refractory Mycoplasma pneumoniae pneumonia with concomitant acute cerebral infarction in a child: A case report and literature review. Medicine (Baltimore) 97: e0103, 2018.

16. Tincani A, Balestrieri G, Allegri F, Cinquini M, Vianelli M, Taglietti M, Sanmarco M, Ichikawa K, Koike T, Meroni P and Boffa MC: Overview on anticardiolipin ELISA standardization. J Autoimmun 15: 195-197, 2000.

17. Kearon C, Akl EA, Ornelas J, Blaivas A, Jimenez D, Bounameaux H, Huisman M, King CS, Morris TA, Sood N, et al: Antithrombotic therapy for VTE disease: CHEST guideline and expert panel report. Chest 149: 315-352, 2016.

18. Monagle P, Cuello CA, Augustine C, Bonduel M, Brandão LR, Capman T, Chan AKC, Hanson S, Male C, Meerpohl J, et al: American society of hematology 2018 Guidelines for management of venous thromboembolism: Treatment of pediatric venous thromboembolism. Blood Adv 2: 3292-3316, 2018.

19. Graw-Panzer KD, Verma S, Rao S, Miller ST and Lee H: Venous thrombosis and pulmonary embolism in a child with pneumonia due to Mycoplasma pneumoniae. J Natl Med Assoc 101: 956-958, 2009. 
20. Chen Y, Huang P, Chen Q, Lin Z and Tian W: Two separated thrombi in deep veins associated with pulmonary embolism after Mycoplasma pneumoniae infection: A case in adolescent female. Transl Pediatr 2: 198-201, 2013.

21. Brown S, Padley S, Bush A, Cummins D, Davidson S and Buchdahl R: Mycoplasma pneumonia and pulmonary embolism in a child due to acquired prothrombotic factors. Pediatr Pulmonol 43: 200-202, 2008

22. Su HY, Jin WJ, Zhang HL and Li CC: Clinical analysis of pulmonary embolism in a child with Mycoplasma pneumoniae pneumonia. Zhonghua Er Ke Za Zhi 50: 151-154, 2012 (In Chinese).

23. Wei H, Chang Y and Lu S: A case report of pulmonary embolism associated with Mycoplasma pneumoniae pneumonia. Zhonghua Er Ke Za Zhi 53: 143-144, 2015 (In Chinese).

24. Zhuo Z, Li F, Chen X, Jin P, Guo Q and Wang H: Mycoplasma pneumonia combined with pulmonary infarction in a child. Int J Clin Exp Med 8: 1482-1486, 2015.

25. Qin Y, Wang H, Wang Y, Zhang W, He L and Liu C: Mycoplasma pneumoniae pneumonia complicated with pulmonary embolism in children: A case report. J Clin Pediatr 37: 765-768, 2019 (In Chinese).

26. Zhang J, Liu F, Guo C, et al: A report of 2 cases of pediatric refractory Mycoplasma pneumonia complicated with pulmonary embolism. Chin J Pract Pediatr 34: 1043-1045, 2019 (In Chinese). doi: 10.19538/j.ek2019120617.

27. Dijl FN, Curtin J, Lord D and Fitzgerald DA: Pulmonary embolism in children. Paediatr Respir Rev 13: 112-122, 2012.

28. Simmons BP and Aber RC: Mycoplasma pneumoniae pneumonia. Symptoms mimicking pulmonary embolism with infarction. JAMA 241: 1268-1269, 1979.

29. Li T, Yu H, Hou W, Li Z, Han C and Wang L: Evaluation of variation in coagulation among children with Mycoplasma pneumoniae pneumonia: A case-control study. J Int Med Res 45: 2110-2118, 2017
30. Narita M: Pathogenesis of neurologic manifestations of Mycoplasma pneumoniae infection. Pediatr Neurol 41: 159-166, 2009.

31. Sotgiu S, Pugliatti M, Rosati G, Deiana GA and Sechi GP Neurological disorders associated with Mycoplasma pneumoniae infection. Eur J Neurol 10: 165-168, 2003.

32. Nagashima M, Higaki T, Satoh $H$ and Nakano T: Cardiac thrombus associated with Mycoplasma pneumoniae infection. Interact Cardiovasc Thorac Surg 11: 849-851, 2010.

33. Senda J, Ito M, Atsuta N, Watanabe H, Hattori N, Kawai H and Sobue G: Paradoxical brain embolism induced by Mycoplasma pneumoniae infection with deep venous thrombus. Intern Med 49: 2003-2005, 2010.

34. Ascer E, Marques M and Gidlund M: M pneumoniae infection, pulmonary thromboembolism and antiphospholipid antibodies. BMJ Case Rep 2011: bcr1220103561, 2011.

35. van Es N, van der Hulle T, van Es J, den Exter PL, Douma RA, Goekoop RJ, Mos IC, Galipienzo J, Kamphuisen PW, Huisman MV, et al: Wells rule and d-Dimer testing to rule out pulmonary embolism: A systematic review and individual-patient data meta-analysis. Ann intern Med 165: 253-261, 2016.

36. Konstantinides SV, Barco S, Lankeit $M$ and Meyer G: Management of pulmonary embolism: An update. J Am Coll Cardiol 67: 976-990, 2016.

37. Moore AJE, Wachsmann J, Chamarthy MR, Panjikaran L, Tanabe Y and Rajiah P: Imaging of acute pulmonary embolism: An update. Cardiovasc Diagn Ther 8: 225-243, 2018.

(c) (1) () $($ This work is licensed under a Creative Commons (c) ${ }_{\mathrm{EY}}$ NO NO Attribution-NonCommercial-NoDerivatives 4.0 International (CC BY-NC-ND 4.0) License. 